\title{
1. Accelerators: characteristics, trends and the new entrepreneurial ecosystem
}

\author{
Israel Drori and Mike Wright
}

\section{INTRODUCTION}

The emergence of accelerators is a notable development in the landscape of new entrepreneurial ecosystems, which include social media, data analytics and internet-based business applications. Recently, accelerators have drawn the attention of entrepreneurship scholars who, examining accelerators' characteristics (Adkins, 2011; Fishback et al., 2007), distinguish them from incubators (Barbero et al., 2014; Hausberg and Korreck, 2017; Hoffman and Radojecich-Kelly, 2012; Isabelle, 2013; Mian et al., 2016). There is also a growing body of scholarship that has sought to develop a taxonomy (Dempwolf et al., 2014) and examine accelerators as a part of emerging ecosystems (Sparks, 2013).

Accelerators in their current forms are part of a relatively recent but rapidly growing phenomenon. They encompass a wide range of organizational types, private and public, aimed at enhancing the capabilities of start-ups through educational programming and processes (Clarysse et al., 2015; Pauwels et al., 2016). While engaging in growing individual start-ups, accelerators are developing new ecosystems and fostering communities of innovation. Thus, accelerators can affect the economy and society in multiple ways, far beyond the direct effects they may have on start-up performance, including influencing the rate and distribution of innovations and the flow of entrepreneurial knowledge and new ideas within and across industrial sectors and countries. Furthermore, various types of accelerators are the source of a wide range of innovations in different fields, such as high-tech, green technology, urban development, transportation, e-commerce, social media and energy.

In this sense, Cohen and Hochberg (2014) provide a "narrow" definition by conceiving accelerators as an organizational form that creates a process for training and exposing start-ups to their ecosystem, in other words, 
"a fixed-term, cohort-based program, including mentorship and educational components, that culminates in a public pitch event or demo day" (p. 4). However, such a narrow definition ignores the essential functions of accelerators related to their modus operandi, objectives, participants and ecosystem. Accordingly, we offer the following definition:

\begin{abstract}
An accelerator is a generic organizational form that aims to stimulate entrepreneurship. It is structured to provide an intensive, limited-period educational program, including mentoring and networking for the cohort of start-up participants selected for each program, to improve their ability to attract investment following the demo day at the end of the program. Accelerators are organizations that serve as gatekeepers and validators of promising business innovations through their embeddedness in their respective ecosystems and, thus, take an active and salient role in socio-economic and technological advancement.
\end{abstract}

In this chapter, we first describe the variety of structures, processes and outcomes characterizing accelerators, based on our field research in accelerators across Europe, Israel and the US, and follow with an overview of the book, concluding with a summary of how accelerators are the building blocks of the new economy's innovative ecosystems.

\title{
TRENDS IN ACCELERATORS
}

Internationally, there has been a rapid growth in the number of accelerators in recent years. In the US, the increasing trend identified by Hochberg (2016) up to 2013 has continued through 2015 (Hathaway, 2016) (Figure 1.1). Bone et al. (2017) found that at the end of 2016 there were 205 incubators, 163 accelerators, 11 pre-accelerators, 7 virtual accelerators and 4 virtual incubators active in the UK.

The growth of accelerators has been especially marked in certain cities. Particularly notable is the continued increase in the annual number of accelerators founded in London and hence in the cumulative number of accelerators (Figure 1.2a). In contrast, there has been a decline in the annual number of accelerators created in Paris, although the total cumulative number of accelerators present is still increasing. This number surged in 2017 triggered by the opening of Station F, noted as the world's largest startup facility (Figure 1.2b). In Berlin, after a rapid rise in 2015, there appears to have been a sharp drop in the number of accelerators created in 2016 (Figure 1.2c). The environment for the development of accelerators in these cities is discussed in more detail in Chapter 8. A rapid increase in other cities is also evident, including in emerging economies. For example, Goswami et al. (2018) note that while the first accelerator in Bangalore, 


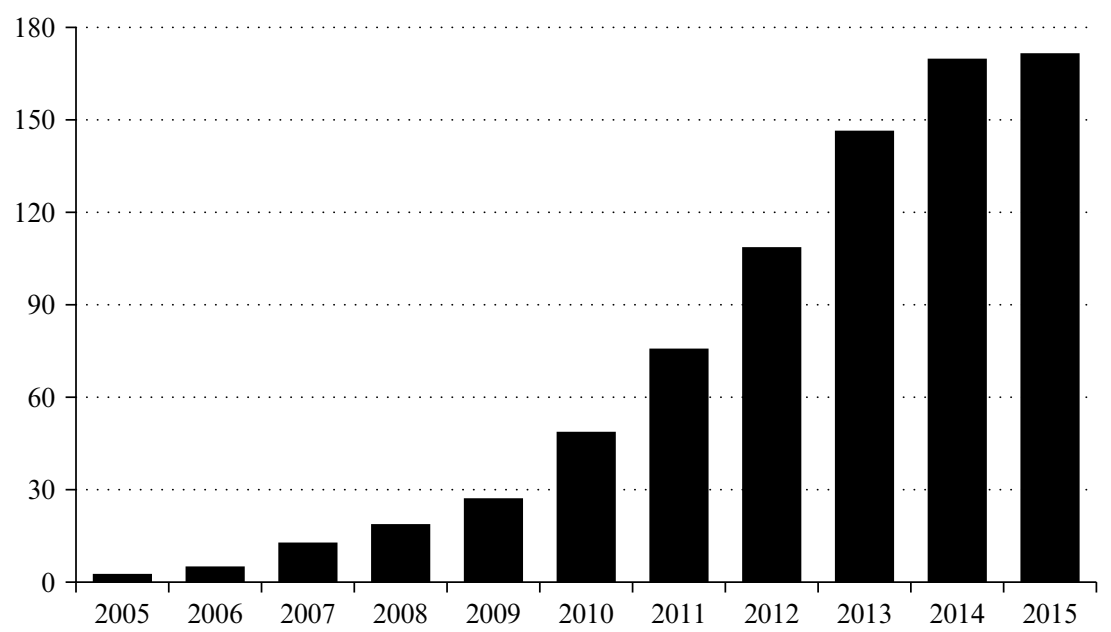

Source: Hathaway (2016). Based on Pitchbook data.

Figure 1.1 US accelerator pool by year

India was launched in 2008, there were 14 accelerators by the end of 2016, four of which were created from April through August 2016.

Because of the fluidity in defining accelerators and their different characteristics as outlined below, estimating their numbers can be challenging. For example, estimates of the number of active accelerators in Israel for 2015 vary from more than 260 (http://www.ivc-online.com) to a more conservative estimate of 90 (for detailed information about accelerators in Israel see: http://www.geektime.com/2016/05/25/do-israeli-accelerators-and-incuba tors-produce-successful-startups-heres-our-full-report/). Accelerators in Israel are of different types: corporate (such as IBM, Coca-Cola, Tyco, AOL, Orange Deutsche Telekom, Intel, Samsung, Yahoo), social, public (government, municipality, academic or kibbutzim affiliation), non-governmental and venture capital (VC) affiliated (such as JVP or Vertex). Some accelerators are joint ventures with other countries (e.g. TechCode, China).

\section{CHARACTERISTICS OF ACCELERATORS}

Accelerators are a unique organizational form; their structure and operational process is relatively basic and requires "lean" managerial personnel and resources. The essence of the accelerating program is extensive 


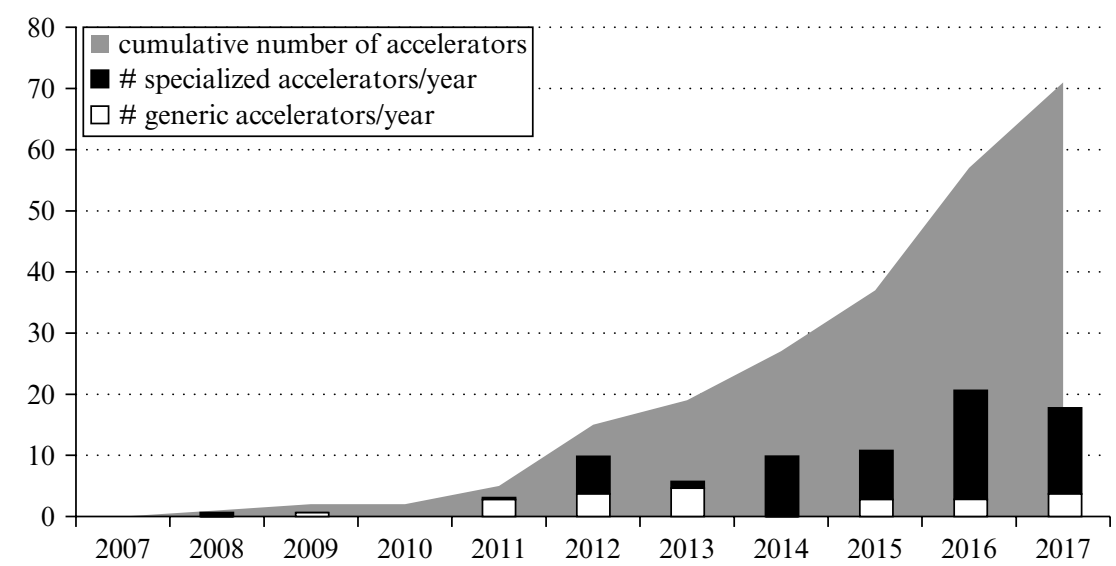

Source: Authors' calculations.

Figure 1.2a Accelerators in London

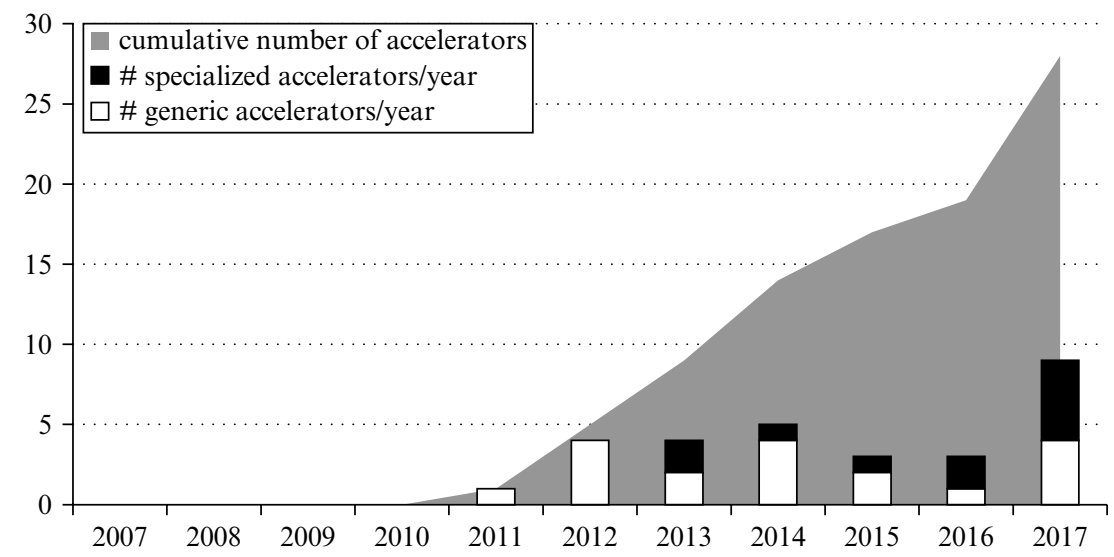

Source: Authors' calculations.

Figure 1.2b Accelerators in Paris

training on entrepreneurship-related issues, along with preparation for a demo day, although as we shall see not all accelerators have a formal demo day. It is "a crash course on entrepreneurship, 300 intensive hours, and it reminds me of an exclusive MBA" claims one of the participants we interviewed (in March 2016). However, accelerators play an important role not 


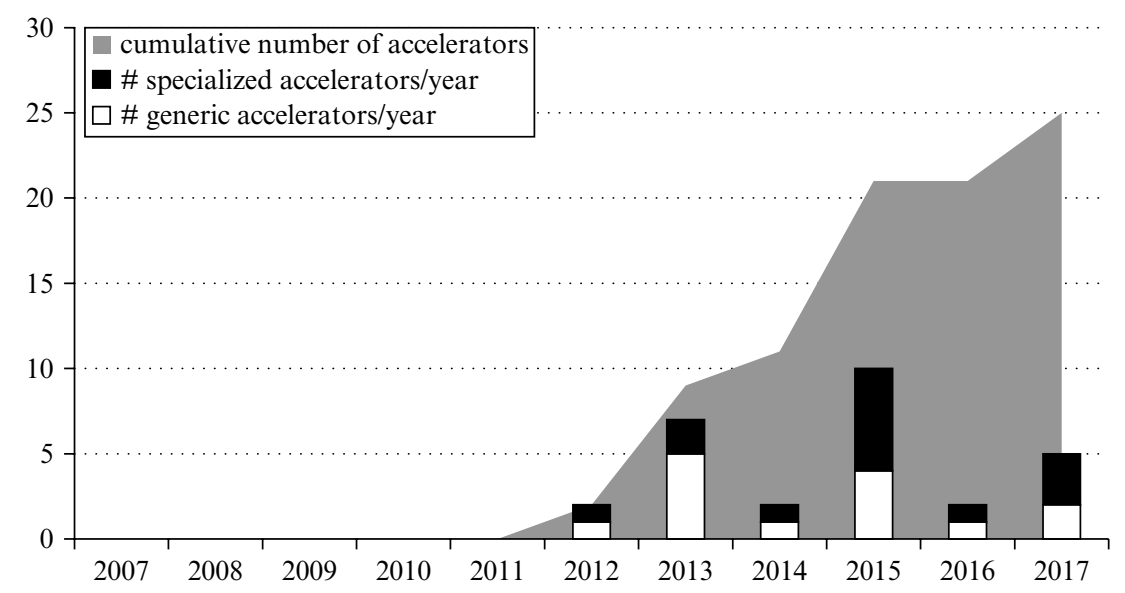

Source: Authors' calculations.

Figure 1.2c Accelerators in Berlin

only in developing the start-ups participating in their respective programs but also in influencing the entrepreneurial ecosystem. In this sense, accelerators serve as brokers, linking start-ups with the environment. There are two features of brokerage. The first, internal, feature is associated with the accelerator's program, mainly outsourcing of expertise and mentoring. The second, which is external, is related to the networking function and the accelerator's role in shaping the innovation and the entrepreneurship landscape of its sector.

The following sections are devoted to the analysis of the dominant characteristics of accelerators. First we analyze accelerators, using the lens of a temporary organization within a permanent organization. This is followed by reviewing accelerators' internal characteristics, mainly structure, ownership and process, and then outlining external characteristics, including accessibility, validity and legitimacy.

\section{ACCELERATORS AND TEMPORARY ORGANIZATION}

Whereas accelerators are usually owned or affiliated with different types of organizations, public or private, their "modus operandi" is highly influenced by the temporal nature of the process associated with their offering to start-ups. When considering the traditional understanding 
of the temporal aspect of organization, indeed, accelerators are organizing temporary activity as part of a permanent organization. However, in understanding the ideational, strategic and operational nature of accelerators, it is useful to view accelerators using a temporal organization lens (Bakker et al., 2016). Recently, Burke and Morley (2016) defined temporary organizations as "a temporary bounded group of interdependent organization actors, formed to complete a complex task" (p. 1237). Using this definition, we present two main features that are the core of the accelerator form of organizing, and their characteristics can best be understood by the temporary dimension of accelerators. These features are (1) participants and (2) organization. Both could be beneficial for further research on accelerators using a temporary organization perspective.

1. Participants Understanding the core meaning of participation in an accelerator program entails a recognition by the start-ups that they are participating in an educational-skill-oriented program which is limited in time. It is a curricula-based program designed to provide specific knowledge pertaining to managing and operating a newly founded firm, including issues beyond technology or product, such as accounting, recruiting, legal aspects of intellectual property, teamwork or consumer behavior. The other distinct offerings of the accelerator, which are also temporary in nature, are mentoring and exposure to varied communities of investors and other stakeholders that have the capacity to facilitate growth of new firms (e.g. accounting firms, established companies). The main thrust of participating in an accelerator program is the idea that effective learning and knowledge is linked to entrepreneurship and technology and is limited in time. Furthermore, selected start-ups accept the leadership and the requirements of the accelerator's management. The nature of participation raises many empirical and theoretical issues, including what is the lasting contribution of the start-ups to the accelerator's educational program? What is the relevance of the program to individual start-ups, particularly in cases of diverse selection in terms of field or technology? What is the effect of the limited time spent in the program and the opportunity cost for the individual start-up? Another aspect of temporality related to the participants is the nature of inter-team relations. Much work on teams in start-ups has drawn on the idea of changing team composition, division of tasks and expertise in accordance with the start-up's progression in its business development (e.g. Drori et al., 2009; Vanaelst et al., 2006). Studying accelerators may provide opportunities to explore neglected aspects of temporary organization, such 
as questions related not only to internal start-ups' team dynamics but also inter-team relations with other firms in the cohort, collaboration or competition, and the development and change of these relations over the entire duration of the program.

2. Organizations The fact that most accelerators are affiliated with a permanent organization raises a series of issues stemming from the potential conflict and tension between the accelerator and its "owner" organization. A key issue is associated with the degree and the scope of autonomy versus embeddedness (Burke and Morley, 2016). Our ongoing research on the different types of accelerators reveals a variety of strategies pertaining to the degree of ownership and the objectives of the owner organization. For example, we found that accelerators that are part of non-governmental organizations (NGOs) whose objectives are related to general societal issues, such as fostering innovation or economic development, enjoy a wide degree of autonomy in terms of criteria of selection, content development, structure and operation of the program. In contrast, accelerators that are part of a distinct public sector organization, such as a municipality, or are financed by corporations or VC, are limited in their autonomy. To a certain degree, they are embedded within their parent organization. This is despite the fact that the accelerator is an independent unit in terms of management, value proposition or service, and is not an integral part of the core activities of the parent organization. However, the accelerator could serve as a hotbed for innovation related to the parent organization's services and technology. Embeddedness also implies that the accelerator is fully financed by the firm that appointed its management and usually allocates space within the firm boundaries (e.g. accelerators affiliated with IBM, Microsoft or Coca-Cola). Thus, accelerators as organizational settings present a promising opportunity to research the fundamental issues of autonomy and embeddedness that mark the relationship between permanent and temporary organizations.

\section{Accelerators' Internal Characteristics}

\section{Structure}

Asked to describe the essence of accelerators as organizational structures designed to host and grow start-ups, an experienced accelerator manager replied: 
An accelerator is first and foremost a kind of school for entrepreneurs. An accelerator is organized in such a way that it provides the pragmatic knowledge and the tools, including learning from one another and linkage to the ecosystem, high-tech and investment experts, lawyers, accountants and program alumni. (Guy, interview with the authors, February 2016)

By and large, the program follows a workshop model. Each meeting is devoted to a different subject, usually elaborated by an expert in the field. One of the accelerator's managers described the educational program as "buffet style": it is up to the participants to pick and choose from predesigned offerings the workshops or activities they consider suitable and helpful.

The topics offered are relatively wide-ranging, especially in accelerators with a diverse cohort in terms of business models and industries. For example, in one of the accelerators we studied, the educational program is essentially divided into two components. The first includes professionally oriented workshops on subjects such as marketing, client relations, branding, business and product cycles, finance and investment, legal issues, the hiring of new employees and technology. These workshops are generic in nature and provide expert knowledge considered relevant for beginner entrepreneurs. The second part of the program involves activities that provide expert skills in the "soft" but crucial areas for start-ups, such as how to build a team, networking, pitching, leadership or dealing with growth of the venture. The latter activities are mainly based on testimonies and lessons-learned narratives of experienced entrepreneurs, combining both failure and success stories. A key component of the entire educational program is an intensive question-and-answer session following each activity, and discussion on the relevance for start-up survival and growth of the subjects covered by the accelerator's educational program.

An important aspect of the program is its fit with the stages and the nature of the start-up. The fit between the program and the start-up depends on the start-up's stage in terms of development of the idea, the technology, the product and the team, in addition to a necessary fit with market and customers. Early-stage start-ups have different requirements, needs and concerns than more mature ventures. In our discussions, founders that consider their companies more mature tend to view a large part of the educational program as redundant and a waste of time. As Yaakov, founder of a start-up in the field of agriculture, claims:

We already have customers and proven technology. We decided to participate in the program because we thought that the benefits would exceed the cost of spending time in class and not with our customers or writing program lines. Even if we learn things, and second-guess, we are not going to change 
our business model. The challenge for the accelerator is to tailor the program to diverse participants, once it decides to adopt a diverse selection policy. (Interview with the authors, May 2016)

Furthermore, the structure of the program dictates an orderly and structured work regimen, which allows the start-ups to focus on problems such as solving technology-related issues or gaining a deeper understanding of their product or market. Participation in the program forces start-up founders to tackle many of their basic assumptions regarding the viability of their business models and to adopt and internalize working and thinking habits and practices that are more conducive to an orderly regime than the chaotic and informal structure characterizing start-ups in their nascent period (Drori et al., 2009).

\section{Ownership and participation}

Accelerators are varied in terms of their owners and affiliations. Generally, they are created and sponsored by either public or private sectors. Moreover, public accelerators (e.g. those operated by municipalities, NGOs, academic institutions) as well as corporate or privately owned accelerators can have heterogeneous participants in terms of life-cycle, content (start-ups with only an idea and a basic team versus those already with customers), technology (concept, demo, beta site), geographical location (city, region, national, international) or investment status (pre-seed, seed, round A). The selected start-ups may be similar, being part of the same industry, mainly horizontally or vertically, but diverse with regard to role and specialization. Yet other accelerators may recruit a cohort of heterogeneous start-ups, with no common ground. These choices depend on the ownership and goals of the accelerator. There are differences between London, Paris and Berlin in terms of the relative importance of generic versus specialized accelerators, but in general a trend over time has been towards more specialized accelerators (Figures $1.2 \mathrm{a}, 1.2 \mathrm{~b}, 1.2 \mathrm{c}$ ).

Although designing a program that fits the needs of a diverse cohort may appear difficult, some programs are generic in terms of the topics they cover, regardless of technology, stage, size or industry of the respective participants. As Uri, the founder of a cybersecurity company participating in Microsoft's accelerator program, explains:

In our batch, we were from different sectors, technology areas and even maturity. This variety makes it difficult to benefit from the concrete insights and help from our peers, but sometimes we got a brilliant idea from someone who is in the tourism business. The program was not tailor made, and we spent precious time on redundant subjects, which are nice to have but not relevant to our field of cybersecurity. (Interview with the authors, January 2016) 
Thus, specialized accelerators, both publicly and privately owned, may select for a certain cohort only those start-ups sharing similar interests (e.g. in the fields of energy, transportation or clean tech). Other accelerators may be more generalized, selecting diverse start-ups for a certain cohort (e.g. fin-tech, e-commerce, clean tech). The type and stage of start-up in terms of idea, technology, team, market and finance selected to their program are major considerations for accelerator owners. The accelerator's selection of start-ups, structure, program and key performance indicators (KPIs) is contingent on the type of ownership and its respective objective. Indeed, a VC-affiliated accelerator is interested mainly in deal flow and eventually financing the most promising participants. Academically affiliated accelerators, on the other hand, are interested in providing relevant entrepreneurial training to students, while NGO-affiliated accelerators may frame a mission that relates to the societal objective of enhancing quality of life.

\section{Process}

Accelerators follow a linear process, starting with the selection of startups, offering educational programming and mentoring, and culminating with a demo day, an event that celebrates the start-up's graduation by pitching to investors and other industry actors. The demo day is also an event that provides an opportunity for the accelerator to reassert its position and legitimacy as an important actor in shaping the new economy's ecosystem through identifying and growing start-ups. As one of the accelerator's managers contends:

The demo day is an opportunity for the start-ups to show what they have, but it is also an opportunity for us to strengthen our relations with our current networks and expand them by inviting new players. It is an opportunity to show the quality of our program and our commitment to build and maintain the Israeli entrepreneurial ecosystem. (Interview with the authors, July 2016)

For commercially oriented accelerators, the process of acceleration serves as a selection tool for the close monitoring of the progress of the start-up and an assessment of its value and potential. In this sense, VC-sponsored accelerators, for example, utilize the program for their investment decision making. The manager of a VC accelerator explains:

We are selecting the best start-up so we first enhance our deal flow. We are providing them with space and "pizza money" and charge eight percent equity. We have an excellent network, but no less important, we are promising the startups that we will invest, ourselves, in the best. I know, this creates pressure and tension, but also healthy competition. Sometimes, the selected start-ups decline to participate because of the consequences of not being selected for investment 
by us, which they think will tarnish their chances to raise money. (Interview with the authors, February 2016)

Cooperation among start-ups is usually considered an asset. In the bylaws of the program of a notable accelerator based in Tel Aviv, Israel, two out of the five items refer to collaboration, including a mandate to "respect one another and be curious and aware of the other's dilemmas and problems, and be a friend to one another." (The first item refers to mandatory attendance and a commitment to "be present, physically and mentally.") Accelerators engage in both explicit and implicit activities to encourage collaboration. Starting with the physical layout, start-ups usually work in a common open space and engage in specialized sessions devoted to providing comments and advising one another on various issues, from technology to marketing. By interacting closely with others, comparing and sharing problems, dilemmas and frustrations, the accelerator provides a supportive environment based not only on the program but also on the peer support of those who have undergone a similar process. One of the accelerator's managers asserts:

Cooperation among the participants is crucial. We start with the selection process. One important criterion is choosing the start-ups. Then, in many of our activities, we work with mixed groups, so each person can bring in his or her insight and knowledge. We encourage the start-ups to help each other in solving practical issues concerning technology, marketing, recruitment or even business strategy. So we can even identify mentors among the start-ups. By encouraging cooperation, we complement the program by sharing the knowledge and experience of the participants. (Interview with the authors, April 2016)

The benefit of cooperation is reiterated by one of the accelerator's participants: "I got more from the other participants than from the mentors and the program. Once you open up and are willing to share your dilemmas with others, you get creative advice" (Eran, interview with the authors, February 2016).

\section{Accelerators' External Characteristics}

\section{Accessibility}

Accelerators evaluate themselves on various KPIs, including their internal objectives and the outcomes of the accelerating program in terms of the sum of its participants' KPIs. Accordingly, the effectiveness of providing access to resources, networking or mentors is contingent upon the start-ups' willingness and ability to use the accelerator's varied offerings. The assumption is that the accelerator provides "optimum accessibility 
to the inputs needed for the successful raising of investment" (accelerator manager, interview with the authors, February 2016), but the benefits are dependent on the start-ups themselves.

A distinct aspect of accessibility is related to the accelerator's objective of encouraging innovative start-ups, disruptive technologies and creative services. In this sense, accelerators perceive themselves as agents of change that feed into the ecosystem stream of innovations and the respective enterprises that attempt to disseminate these innovative products or services, for either profit or non-profit purposes. In particular, corporate accelerators such as those operated by Microsoft or IBM justify the rationale for having an in-house accelerator, in spite of the cost and the status as a non-profit activity, as a link to the dynamic innovative sector of start-ups. The general managers of the Microsoft accelerator claim:

\begin{abstract}
Start-ups are a hotbed for innovations. Through the accelerator's participants, we are able to develop new ideas and technological solutions. We are tapping on potential sources of new innovation and new technology. We are a behemoth, and we sometimes are not linked to the new cool things individual entrepreneurs are doing. Through the accelerator, we have access to innovation and at the same time, we disseminate our cloud technology, the Azure. (Interview with the authors, August 2016)
\end{abstract}

Furthermore, the technological solutions and other lessons learned regarding growing start-ups accumulated by the accelerators are usually disseminated from cohort to cohort. Such accessibility of knowledge makes the accelerator an effective organizational form for growing new firms. As the accelerator accumulates experience and knowledge, it operates more effectively. Dani, an accelerator manager, stated:

We learn from every cohort, always new things in technology, products, marketing or business. This is our asset. Sometimes, it is an automatic understanding of what to do and not to do, or intuition regarding how to develop a promising business plan, and what investor or team member is needed. (Interview with the authors, March 2016)

In sum, accelerators play an important social and economic role as an organizational form that not only identifies opportunities for innovation, but also facilitates their distribution in the environment.

\title{
Validity and legitimacy
}

Accelerators are well-linked organizations within their respective ecosystems. In many cases, accelerators see themselves as the creators of the ecosystem. One of the notable Israeli accelerators in the field of transportation developed a network of all the organizational players that deal with 
transportation and related issues, including the private and public sectors, academia and start-ups. Being in the bridging position enables the accelerator to be one of the key actors that shape the agenda of the sector, to generate important information that helps in designing its activity and to provide effective validation for the start-ups that graduate from its program.

An important aspect of legitimacy is the standing and reputation of the accelerators. In numerous discussions with start-ups' founders, we have found that the issue of the selection of accelerators strongly influences their decision to apply and participate in a given accelerator's program. For example, being selected by the Microsoft accelerator implies high prospects for exposure to the best investors; it is a door opener. As one of the founders explains:

Microsoft provides you not only material resources, their Azure, but also bestows on us their reputation, the possibility to be acquired by them and to be respected as an important player in the industry.

Participation in the program is like a "Kosher" stamp. It signals to everyone that you are an innovative and worthy start up. (Rami, interview with the authors, May 2016)

Thus, a connection to Microsoft's reputation, standing and network is seen as an asset that the start-up acquires, in addition to its business idea, product, technology or service; an asset that bestows validity and legitimacy.

Another important factor with regard to legitimacy is the use of the accelerator's close network. Inbal, the founder of a renowned accelerator in Tel Aviv, explains: "We are not a local accelerator. We are offering to the start-up a global network, by using our alumni [of the specific technological army unit that founded the accelerator], which are operating in places such as Silicon Valley or Berlin" (Interview with the authors, January 2016). Indeed, a distinct class of accelerators could be termed "network accelerators," whose main objective is to serve as brokers of a kind, a link between the start-up and the investors. Their legitimacy resides in their ability to match appropriate investors to the start-ups and, at the same time, to benefit from "finders' fees."

An accelerator's internal design may also be used as a tool in its legitimation strategies. Specifically, incorporating distinguished partners in an industry as sponsors of a designated cohort enhances both internal and external legitimacy, among start-ups and external organizations, respectively.

Validation is considered a by-product of the acceleration process. It embodies various aspects. The first is validation of the start-up's product, market or technology. Some accelerators search for ideas and teams, and provide the platform - work space and some resources. The accelerator 
may be very active in assisting the start-up in developing the business model and/or the company itself. In this sense, accelerators serve as coaches, steering the start-up towards obtaining investment. Another form of validity concerns the reputation of the accelerator as a hub for promising start-ups. Successful accelerators, claims one of our interviewees, a journalist who covers the Israeli high-tech industry, "develop a reputation for selecting the best start-up in terms of ideas and teams and upgrading their abilities considerably. In this way, they signal to investors that their start-ups are dependable and promising. In this way, accelerators provide a quality assurance stamp" (Interview with the authors, May 2016). Thus, accelerators signal to the ecosystem that the start-up has undergone an adequate selection process, in addition to an educational program that improves its capabilities.

The role of validator puts pressure on accelerators to focus their program on those aspects important to investors, sometimes at the expense of their other mission. For example, an accelerator's manager testifies:

I have to compete with other accelerators for my position as a leading validator, so I align my KPI to fit the market need, devoting, for example, too many sessions to branding and less to other social activities. Eventually, I decided to limit my role as validator only for early stage start-ups. (Interview with the authors, February 2016)

Accelerators are organizations that influence their ecosystem through the implementation of programs aimed at preparing and providing startups with business and investment opportunities. Lean organizations in terms of structure and management, accelerators focus on the strategic idea that their programs do not stand alone; their quality and impact are dependent upon the accelerator's standing and embeddedness in its ecosystem. A thick network of mentors, sponsors, alumni and other key players, such as investors or specialized consultants, enables accelerators not only to attract promising start-ups, but also to maintain a reputation as validators. Thus, an effective accelerator is considered a bridge between those who initiate an enterprise (founders of start-ups) and those who can help an enterprise take off (investors, adopters of technology or product). Accelerators usually execute a linear program, starting with the selection of start-ups and continuing with intensive learning curricula that are highly diverse in terms of content and format. An integral part of the program is mentoring on the one hand and peer cooperation on the other. The official acceleration program ends with a demo day, a pitch, an event that exposes both the start-ups and the accelerator to potential investors and other gatekeepers of emerging ecosystems. Although small in size and limited in its objectives, the accelerator is an enterprise in its own right, an organization that specializes in learning and connecting through both its 

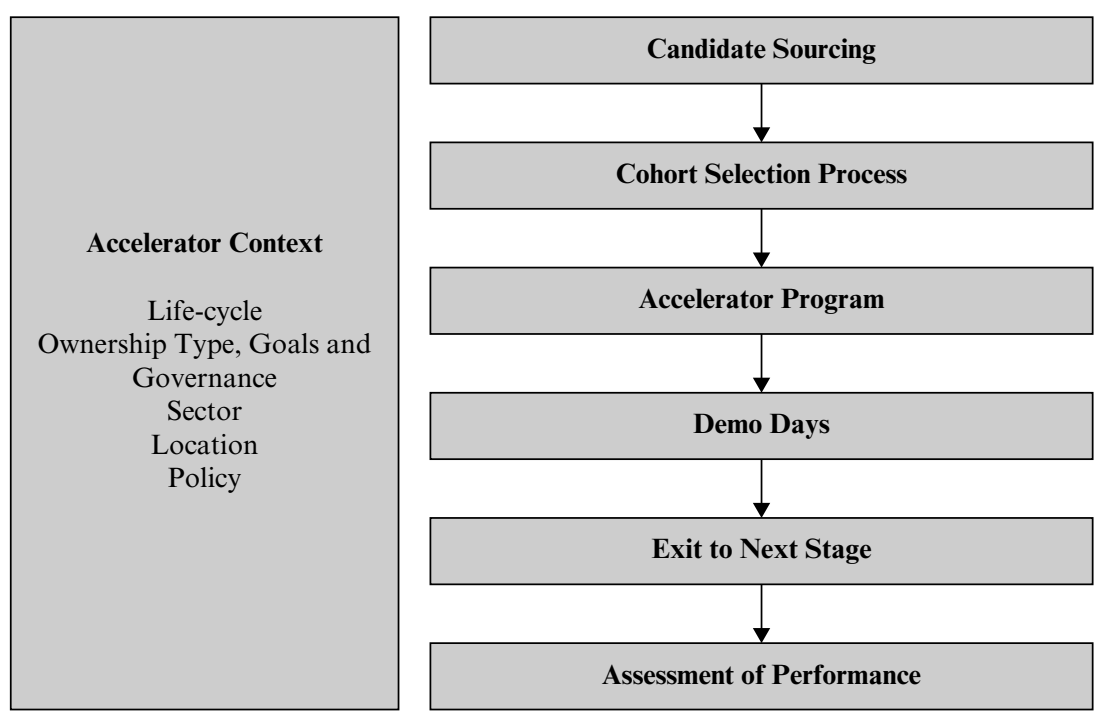

Figure 1.3 Accelerator process life-cycle

own knowledge and output and its ability to harness actors and forces in the environment for the purpose of facilitating and simplifying the work of entrepreneurs.

\section{STRUCTURE OF THE BOOK}

This book is structured on the basis of the life-cycle of accelerator programs for new ventures (Figure 1.3). This life-cycle starts with the means by which potential candidate ventures are sourced; the selection process for each cohort, the nature of the accelerator program, the nature and role of demo days; issues relating to the exit from the program and the next stage in the venture's life-cycle, including seeking investors; and the assessment of the performance of the ventures in each cohort and of the accelerator itself. These elements in the life-cycle process are analyzed in the context of how they are impacted by different dimensions of context.

\section{Acceleration Themes}

The chapters comprising the principal content of this book draw on evidence worldwide from developed and developing economies, including the 
US, UK, France, Germany, the EU, Australia and Chile. They cover the following themes.

In Chapter 2, Matthias Wenzel and Jochen Koch develop conceptual arguments on what and how accelerators accelerate. Based on the literature on strategy process, they draw two conceptual distinctions that relate to the object of acceleration (strategic core versus strategic periphery) and the processual patterns of acceleration (forwarding versus leaping). They then use these distinctions to conceptualize and discuss four ways in which accelerators can accelerate the business development of new ventures. They argue that start-up acceleration seems to be largely aimed at forwarding and leaping the strategic periphery instead of the strategic core. This chapter extends the emerging literature on accelerators by making the case for considering and examining start-up acceleration as a process and offering a more specific and nuanced understanding of how it occurs.

In Chapter 3, Laurens Vandeweghe and Trent $\mathrm{Fu}$ provide a micro-lens on business accelerators' governance practices by examining how accelerators manage their external networks and internal organizations to achieve their organizational goals. As such, they look both at how accelerators coordinate autonomous stakeholders such as partners, investors and portfolio companies within a network form of governance and at how accelerators are monitored and controlled internally to preside over the day-to-day activities by organizational governance mechanisms. They classify accelerators based on their sponsorship (i.e. corporate, private, public), indicating that such sponsorship differences influence the ways in which accelerators are governed both from an external-network perspective and from an internal organizational perspective, and illustrate this by applying this dual perspective to the cases of AppCampus, Techstars and Start-Up Chile.

Ronit Yitshaki and Israel Drori focus in Chapter 4 on the important aspect of the accelerators' design structure and operation that concerns mentoring. By mentoring they refer to the process of learning and coaching provided by the accelerators to their participating start-ups by groups of experts with relevant knowledge and experience in founding and managing new ventures. Using detailed interview and observational data from Israel, they show that the mentoring process provides a bridge between the accelerator, the start-up and the ecosystem through the mentor's internal (within the start-up) and external (within the ecosystem) position. They suggest that mentorship is a complex process composed of both altruistic and interest-based motivations and processes, represented along a continuum starting as part of the accelerator's educational program, and either ending with the end of the program or leading to a transformation to a partnership. Accelerators play an important role in recognizing mentors 
who are relevant to the ecosystem while providing mentors with opportunities to invest and learn about the ecosystem, enabling them to conduct informal due diligence prior to investing as business angels. They identify four distinct aspects that characterize the mentoring process: (1) setting up strategy and priorities, (2) revealing marketing opportunities, (3) structuring organizational processes, and (4) expanding ventures' social capital.

Chapter 5 by Michael Leatherbee and Juanita Gonzalez-Uribe addresses issues relating to the selection of entrepreneurs and their ventures for entry into accelerators. Commonly, accelerators select start-ups among a broader group of applicants. The assumption is that through the selection process, accelerators are able to discriminate between high- and low-potential startups. Thus, the expectation is that accelerators are an effective medium for capturing the upside potential of the select few start-ups that promise to deliver the highest value in the future. That upside potential may be materialized through attractive equity investments or increased socio-economic development, depending on the mission of the accelerator. Typically, the selection process relies on a set of objective criteria predetermined by the accelerator, which are applied by one or more entrepreneurship experts who act as judges or evaluators of the applicant pool. First the authors describe the different selection stages and methods typically managed by business accelerators. They then go on to explore the multiple important issues that must be taken into account when designing and managing selection processes. Comprehending these issues may help to understand the challenges and limitations of current selection methods, and to avoid potential pitfalls and unintended consequences.

Chapter 6 by Leatherbee and Gonzalez-Uribe examines KPIs in accelerators. KPIs can help gauge the health and progress of business accelerators. Their use is fundamental for learning how to improve organizational and programmatic effectiveness, and for building a data-driven shield from program skeptics. However, figuring out which indicators are best, how to develop them and what phenomenon they are actually reflecting is a nontrivial exercise. The authors provide an overview of different KPIs that can be used by accelerator stakeholders. By explaining their value, limitations, methods of construction and potential pitfalls, they aim to provide accelerator stakeholders with a toolkit for building an effective KPI dashboard.

Policymakers are one of the key stakeholders in the entrepreneurial ecosystem in which accelerators are embedded. We present two chapters covering policy issues. In Chapter 7, Iris Vanaelst, Jonas Van Hove and Mike Wright, after a brief review of US policy towards accelerators, focus on how EU policy engages with different stakeholders in order to support accelerator activity within the EU area. The EU interactions with accelerators are threefold: (1) the EU supports the setup of accelera- 
tor networks to create momentum for accelerators to meet and exchange experiences, expertise and knowledge (e.g. Accelerator Assembly); (2) the EU supports and funds accelerator programs (e.g. EU-XCEL-European Virtual Accelerator, IoT Accelerator Programme, Copernicus Accelerator Programme); and (3) accelerators serve as intermediaries between the EU and start-ups looking for funding (e.g. European Pioneers) in addition to the small and medium-sized enterprise (SME) funding instruments of the EU (e.g. SME Instrument). Interesting examples of each of these initiatives are discussed in more detail.

In Chapter 8, Van Hove, Vanaelst and Wright explore the differences across countries and regions in accelerator support programs in order to improve understanding of the continuously evolving accelerator landscape and concomitant activities by complementary actors within an ecosystem community. They emphasize the role of institutional intermediaries in shaping the ecosystem through the preservation of the aligned interests between policymakers and practitioners. Reviewing the evolution of the landscape of policy support for accelerators across national and regional levels, with particular emphasis on the UK context, they outline the challenges accelerators face and which policymakers need to take into account. They conclude with policy implications and recommendations focusing on how the ecosystem community can meet such challenges in order to spur ecosystem development and fuel the next generation of start-ups.

In Chapter 9, Martin Bliemel, Saskia de Klerk, Ricardo Flores and Morgan Miles review the historic context in which an accelerators industry emerged in Australia in parallel with policy development to indirectly support start-ups. Their study spans over three decades of policies aimed at supporting innovative high-growth ventures. Early attempts at stimulating an incubator industry failed. The authors focus on the most recent years, during which accelerators initially emerged in Australia in the absence of policy tailored to the accelerator business model, followed by the design and implementation of a A \$23 million national Incubator Support Programme.

Finally, in Chapter 10, Massimo Colombo, Cristina Rossi-Lamastra and Mike Wright set out an agenda for future research on accelerators. They suggest that the agenda for future research is multi-leveled, involving the accelerators and their programs as well as the firms, entrepreneurs and mentors involved. As accelerators operate in a range of different contexts, a future research agenda needs to consider the dimensions of these contexts. As accelerators are a rapidly evolving phenomenon, there is also the imperative to consider the time dimension with respect to life-cycle issues. 


\section{CONCLUSIONS}

In sum, accelerators serve as an organizational type that mobilizes both internal and external resources to enhance the innovation capabilities of start-ups and expose these innovations to markets and institutions. This mission of accelerators, of growing start-ups and fostering ecosystems of innovation, has placed them at the forefront of providing support for innovations in which new knowledge, products or services emanate from speculative ideas and entail risky execution. Studies on the innovation emerging from startups tend to focus either on the dynamics of creating innovation or on the constraints on innovation stemming from both organizational and market needs. However, the ability of start-ups to create innovations is dependent on ties and networks that connect communities of start-ups. Furthermore, most existing accounts of the emergence of innovation in start-ups provide little explanation about the actual process and how to enhance innovation through the creation of communities. This book seeks to fill the gap in understanding in how new innovative ecosystems emerge. We have compiled chapters on accelerators, organizational forms that serve as facilitators and brokers that shape the path and the pace of growing start-ups into viable organizations at the forefront of our rapidly changing society.

\section{REFERENCES}

Adkins, D. 2011. What are the new seed or venture accelerators? NBIA Review. Available at http://www.nbia.org/resource_library/review_archive/0611_01.php (accessed April 13, 2017).

Bakker, R.M., DeFilippi, R.J., Schwab, A. and Sydow, J. 2016. Temporary organizing: promises, processes, problems. Organization Studies, 37(12), 1703-1719.

Barbero, J.L., Casillas, J.C., Wright, M. and Garcia, A.R. 2014. Do different types of incubators produce different types of innovations? Journal of Technology Transfer, 39(2), 151-168.

Bone, J., Allen, O. and Haley, C. 2017. Business incubators and accelerators: the national picture. BEIS Research Paper \#7. BEIS/Nesta.

Burke, C.M. and Morley, M.J. 2016. On temporary organizations: a review, synthesis and research agenda. Human Relations, 69(6), 1235-1258.

Clarysse, B., Wright, M. and Van Hove, J. 2015. A Look Inside Accelerators. London: Nesta.

Cohen, S. and Hochberg, Y. 2014. Accelerating startups: the seed accelerator phenomenon (March 30, 2014). Available at SSRN: https://ssrn.com/ abstract $=2418000$ or http://dx.doi.org/10.2139/ssrn.2418000 (accessed November 9, 2016).

Dempwolf, C., Auer, J. and D'Ippolito, M. 2014. Innovation Accelerators: Defining Characteristics among Startup Assistance Organizations. Available at https:// 
www.sba.gov/sites/default/files/rs425-Innovation-Accelerators-Report-FINAL. pdf (accessed April 13, 2017).

Drori, I., Honig, B. and Wright, M. 2009. Transnational entrepreneurship: an emergent field of study. Entrepreneurship Theory and Practice, 33(5), 1001-1022.

Fishback, B., Gulbranson, C.A., Litan, R.E., Mitchell, L. and Porzig, M. 2007. Finding business 'idols': a new model to accelerate start-ups (July 2007). Available at SSRN: https://ssrn.com/abstract=1001926 or http://dx.doi. org/10.2139/ssrn.1001926 (accessed January 23, 2018).

Goswami, K., Mitchell, R. and Bhagavatula, S. 2018. Accelerator expertise: understanding the intermediary role of accelerators in the development of the Bangalore entrepreneurial ecosystem. Strategic Entrepreneurship Journal. DOI:10.1002/sej.1281.

Hathaway, I. 2016. Accelerating growth: startup accelerator programs in the United States. February 17, 2016. Washington, DC: The Brookings Institution. Available at: https://www.brookings.edu/research/accelerating-growth-startupaccelerator-programs-in-the-united-states/ (accessed January 10, 2018).

Hausberg, J.P. and Korreck, S. 2017. A systematic review and research agenda on incubators and accelerators (February 3, 2017). Paper to be presented at the Academy of Management Annual Meeting 2017, Atlanta (GA). Available at SSRN: https://ssrn.com/abstract=2919340 or http://dx.doi.org/10.2139/ssrn. 291 9340 (accessed January 23, 2018).

Hochberg, Y. 2016. Accelerating entrepreneurs and ecosystems: the seed accelerator model. Innovation Policy and the Economy, 16, 25-51. DOI:10.1086/684985.

Hoffman, B. and Radojecich-Kelly, N. 2012. Analysis of accelerator companies: an exploratory case study of their programs, processes, and early results. Small Business Institute ${ }^{\circledR}$ Journal, 8(2), 54-70.

Isabelle, D. 2013. Key factors affecting a technology entrepreneur's choice of incubator or accelerator. Technology Innovation Management Review, February, 16-22. Available at http://www.timreview.ca/article/656 (accessed April 13, 2017).

Mian, S., Lamine, W. and Fayolle, A. 2016. Technology business incubation: an overview of the state of knowledge. Technovation, 50-51, 1-12.

Pauwels, C., Clarysse, B., Wright, M. and Van Hove, J. 2016. Understanding a new generation incubation model: the accelerator. Technovation, 13-24, 50-51.

Sparks, E. 2013. Top trends in state economic development. Washington, DC: National Governors Association. Available at http://www.nga.org/files/live/sites/ NGA/files/pdf/2013/1308TopTrendsinStateEconDevPaper.pdf (accessed April 13, 2017).

Vanaelst, I., Clarysse, B., Wright, M., Lockett, A. and Moray, N. 2006. Entrepreneurial team development in academic spinouts: an examination of team heterogeneity. Entrepreneurship Theory and Practice, 30(2), 249-271. 